20

\title{
Фемтосекундная абсорбционная спектроскопия восстановленной и окисленной форм цитохром $c$ оксидазы: возбужденные состояния и релаксационные процессы в гемовых центрах $a$ и $a_{3}$
}

\author{
(С) И.В. Шелаев ${ }^{1}$, Ф.Е. Гостев ${ }^{1}$, Т.В. Выгодина ${ }^{2}$, С.В. Лепешкевич ${ }^{3}$, Б.М. Джагаров ${ }^{3,}$ \\ ${ }^{1}$ Федеральный исследовательский центр химической физики им. Н.Н. Семенова РАН \\ 119991 Москва, Россия \\ ${ }^{2}$ Научно-исследовательский институт физико-химической биологии им. А.Н. Белозерского, \\ Московский государственный университет им. М.В. Ломоносова, \\ 119991 Москва, Россия \\ ${ }^{3}$ Институт фризики им. Б.И. Степанова НАН Беларуси, \\ 220072 Минск, Беларусь \\ ฯ e-mail: b.dzhagarov@ifanbel.bas-net.by
}

Поступила в редакцию 13.03.2019 г.

В окончательной редакции 13.03.2019 г.

Принята к публикации 11.06.2019 г.

\begin{abstract}
Методом фемтосекундной абсорбционной спектроскопии исследованы возбужденные электронные состояния и внутригемовые релаксационные процессы в окисленной и восстановленной формах митохондриальной цитохром $c$ оксидазы, выделенной из сердечной мышщы быка. Измерения спектрально-кинетических характеристик короткоживущих интермедиатов были выполнены во временном интервале $80 \mathrm{fs}-20 \mathrm{ps}$ после акта фотовозбуждения. Обнаружено, что электронная безызлучательная релаксация энергии возбуждения в геме $a$ как в окисленной $\mathrm{Fe}(\mathrm{III}) a$, так и в восстановленной форме $\mathrm{Fe}(\mathrm{II}) a$ осуществляется последовательно в виде трех процессов, по завершению которых гем $a$ оказывается в основном состоянии с большим запасом колебательной энергии. Последующая колебательная релаксация („остывание гема“) длится несколько пикосекунд. Для восстановленного гема $a_{3}\left(\mathrm{Fe}(\mathrm{II}) a_{3}\right)$ было обнаружено, что электронная релаксация осуществляется в результате двух последовательных стадий, которые сменяются колебательной релаксацией в основном состоянии. Дан анализ механизма и динамики преобразования энергии электронного возбуждения в цитохром $c$ оксидазе.
\end{abstract}

Ключевые слова: цитохром $c$ оксидаза, фемтосекундная абсорбционная спектроскопия, возбужденные электронные состояния, релаксационные процессы, спектральные интермедиаты.

DOI: $10.21883 /$ OS.2019.10.48381.99-19

\section{Введение}

Цитохром $c$ оксидаза (ЦО) является терминальным элементом дыхательной цепи митохондрий и дышащих бактерий. ЦО катализирует четырехэлектронное восстановление молекулярного кислорода до воды. Данная реакция сопряжена с захватом восьми протонов $\left(8 \mathrm{H}^{+}\right)$ с внутренней стороны мембраны, из которых $4 \mathrm{H}^{+}$(субстратные) идут на образование двух молекул $\mathrm{H}_{2} \mathrm{O}$, а другие $4 \mathrm{H}^{+}$(помповые) переносятся на внешнюю сторону мембраны. Общий вид данной реакции представляется следующим образом:

$$
4 \text { cyt } c^{2+}+\mathrm{O}_{2}+8 \mathrm{H}^{+}=4 \mathrm{cyt} c^{3+}+2 \mathrm{H}_{2} \mathrm{O}+4 \mathrm{H}^{+},
$$

где $\operatorname{cyt} c$ обозначает цитохром $c$, который является поставщиком электронов. Суt $c^{2+}$ и суt $c^{3+}-$ соответственно восстановленная и окисленная формы данного гембелка [1-3].

Перенос электронов в ЦО осуществляется четырьмя редокс-центрами: двумя гемами (низкоспиновый гем $a$ и высокоспиновый гем $\left.a_{3}\right)$ и двумя атомами меди $\mathrm{Cu}_{\mathrm{A}}$ и $\mathrm{Cu}_{\mathrm{B}}$. Химическая структура гемов $a$ и $a_{3}$ одинаковая.
Они представляют собой комплекс иона железа с порфириновым макроциклом. В высокоспиновом геме $a_{3}$ атомы $\mathrm{Fe}$ и $\mathrm{Cu}_{\mathrm{B}}$ расположены примерно в 4-5 $\AA$ друг от друга и образуют бинуклеарный каталитический сайт, в котором собственно и происходит восстановление $\mathrm{O}_{2}$ до $\mathrm{H}_{2} \mathrm{O}$. Последовательность этапов переноса электрона может быть представлена в виде следующей схемы:

$$
\text { cyt } c \rightarrow \mathrm{Cu}_{\mathrm{A}} \rightarrow \text { гем } a \rightarrow \text { гем } a_{3} / \mathrm{Cu}_{\mathrm{B}} \rightarrow \mathrm{O}_{2} \text {. }
$$

Несмотря на многолетние интенсивные исследования с привлечением всевозможных физических и химических экспериментальных методов и теоретических подходов механизм обсуждаемого цикла реакций до сих пор до конца не расшифрован.

В настоящей работе мы используем фемтосекундную абсорбционную спектроскопию для обнаружения и изучения возникающих и релаксирующих интермедиатов во временном интервале $80 \mathrm{fs}-20 \mathrm{ps}$. Использование кинетической спектроскопии представляется необходимым, так как принципиально важным моментом является установление путей и скоростей переноса электрона 
внутри данной биоэнергетической системы. Действительно, в процессе функционирования ЦО должны реализовываться как межгемовые, так и внутригемовые переносы электрона между Fe и $\pi$-сопряженной системой порфирина, т. е. переносы $\pi$-электронов на $d$-орбитали и переносы $d$-электронов на низшие незаполненные $\pi$-орбитали. При этом необходимо учитывать, что эти процессы переноса электронов быстропротекающие.

Необходимо отметить, что исследования ЦО исключительно сложны из-за перекрытия спектров поглощения гема $a$ и гема $a_{3}$, что крайне затрудняет определение их индивидуальных спектральных характеристик [4-6]. Как отмечалось ранее, химическая структура гемов $a$ и $a_{3}$ одинакова. Однако атомы железа различаются в них спиновыми состояниями и степенью заполнения координационной сферы. Атом железа в геме $a$ шестикоординирован четырьмя атомами азота пиррольных колец и двумя аксиальными гистидинами, а в геме $a_{3}$ пятикоординирован четырьмя атомами азота и одним гистидином, и имеет свободное координационное положение для присоединения $\mathrm{O}_{2}$. Это положение могут занимать и другие малые лиганды: $\mathrm{CO}, \mathrm{CN}$. Из-за различного заполнения координационной сферы $d$-орбитали в гемах заполняются различным способом, и в результате различаются спиновыми состояниями. Атом железа в геме $a$ является низкоспиновым (спин $S=1 / 2$ в состоянии $\mathrm{Fe}^{3+}$ и $S=0$ в состоянии $\left.\mathrm{Fe}^{2+}\right)$. Fe в геме $a_{3}-$ высокоспиновый (спин $S=5 / 2$ в состоянии $\mathrm{Fe}^{3+}$ и $S=2$ в состоянии $\mathrm{Fe}^{2+}$ ).

Различаются у гемов и спектры поглощения [4-6]. Максимумы поглощения полос Соре в геме $a$ и геме $a_{3}$ расположены соответственно при 426 и $414 \mathrm{~nm}$ и формируют общий пик в спектре при $421 \mathrm{~nm}$ (рис. 1). В длинноволновой области спектра слабый пик при $600 \mathrm{~nm}$. В восстановленной форме ЦО полосы в спектре поглощения перекрываются гораздо сильнее, образуя общий максимум при $444 \mathrm{~nm}$ с короткоживущим плечом при $\sim 425 \mathrm{~nm}$ (рис. 1), который вызван поглощением гема $a_{3}$. Менее интенсивный максимум при $605 \mathrm{~nm}$ обусловлен в основном поглощением гема $a^{2+}[4-6]$.

Таким образом, настоящая работа посвящена изучению внутригемовой энергетики в гемах $a$ и $a_{3}$. Настоящая работа в известной степени продолжает наши предыдущие исследования, результаты которых были представлены в работе [7], посвященной изучению полностью восстановленной формы ЦО, т.е. такой формы, у которой ион железа находится в состоянии $\mathrm{Fe}(\mathrm{II})$, как в геме $a$, так и в геме $a_{3}$. Цель настоящей работы заключалась в изучении, во-первых, полностью окисленной формы ЦО, у которой ионы железа находятся в состоянии $\mathrm{Fe}(\mathrm{III})$, во-вторых, в проведении дополнительных исследований восстановленной ЦО в спектральном диапазоне $425 \pm 5 \mathrm{~nm}$. В этом случае мы исходили из следующих соображений. Поглощение в этой спектральной области в большей степени принадлежит гему $a_{3}$, поскольку согласно реконструкции абсолютного спектра восстановленного гема $a$, основанной

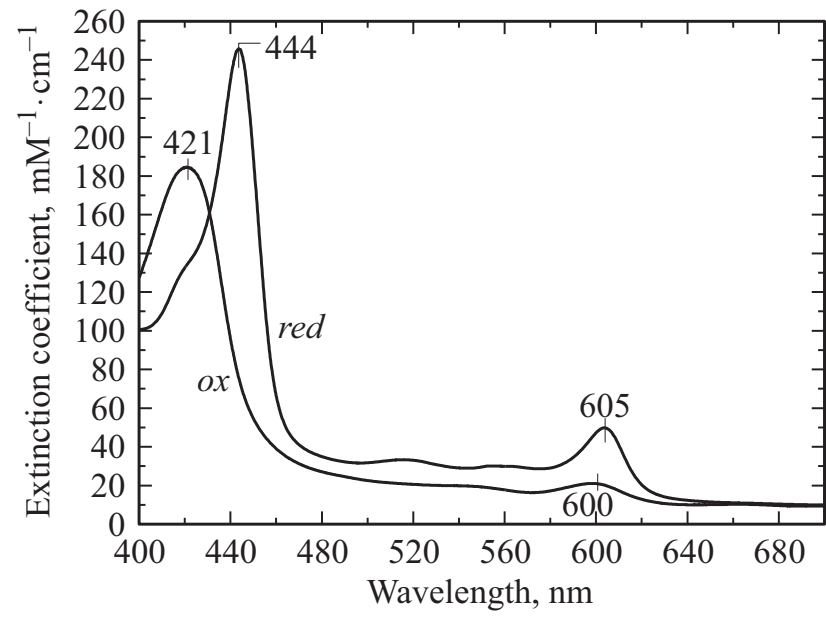

Рис. 1. Спектр поглощения восстановленной (red) и окисленной (ох) цитохром с оксидазы.

на спектральном сдвиге, вызываемом ионами $\mathrm{Ca}^{2+}[5]$, его максимум $447 \mathrm{~nm}$ сдвинут в более длинноволновую область по сравнению с максимумом $443 \mathrm{~nm}$, характеризующим полосу поглощения восстановленной ЦО (рис. 1), которая отражает поглощение обоих гемов. Таким образом, появляется возможность в ходе исследований получить индивидуальные спектральнокинетические характеристики возбужденных состояний гемов $a$ и $a_{3}$ для восстановленной формы ЦО.

\section{Экспериментальная часть}

Объекты исследований. Цитохром с оксидазу выделяли из сердечной мышцы быка по методу, описанному в работе [8] (модифицированный метод Фаулера и др. [9]), следуя протоколу, любезно предоставленному доктором Мусатовым (Институт экспериментальной физики, Словацкая академия наук, г. Кощице, Словакия). Основные измерения выполнялись с образцами ЦО с концентрацией $50 \mu \mathrm{M}$, которая определялась на основании разностного спектра поглощения (восстановленный дитионитом образец минус окисленный) с использованием значения $\Delta \varepsilon=27 \mathrm{mM}^{-1} \mathrm{~cm}^{-1}$, характерного для двух длин волн $605 / 630 \mathrm{~nm}$. В качестве буферного раствора использовался $0.1 \mathrm{M}$ Tris-Hepes буфер $\mathrm{pH} 8.0$, 0.05\% ДДМ (додецил мальтозид), $0.1 \mathrm{mM}$ ЭГТА (этиленбис(оксиэтилен-нитрило)-тетрауксусная кислота). Спектры поглощения для двух изучаемых в работе форм цитохром $c$ оксидазы представлены на рис. 1.

Фемтосекундная установка. Разрешенные во времени дифференциальные спектры наведенного поглощения $\Delta A(\lambda, t)$ измерялись методом „возбуждение-зондирование“ с помощью фемтосекундной абсорбционной установки, подробно описанной в работе [10]. Для возбуждения объектов исследования использовались гауссовы импульсы с частотой следования $60 \mathrm{~Hz}$, длительностью $23 \mathrm{fs}$, с энергией $350 \mathrm{~mJ}$, с максимумом 
излучения при $600 \mathrm{~nm}$. Эти импульсы фокусировались в пятно диаметром $180 \mu \mathrm{m}$. Импульсы белого континуума фокусировались в пятно диаметром $120 \mu \mathrm{m}$ и использовались в качестве зондирующего света. Поляризации импульсов накачки и зондирования были ориентированы под магическим углом 54.7 друг к другу. Суперконтинуум диспергировался с помощью полихроматора (Action SP300) и регистрировался с помощью CCD-камеры (Roper Scientific SPEC 10). Коррекция нулевого времени задержки между импульсом накачки и спектральной компонентой $\lambda$ импульса зондирования выполнялась, следуя процедуре, описанной ранее [11].

Дифференциальные транзиентные спектры поглощения $\Delta A(\lambda, t)=A(\lambda, t)-A_{0}(\lambda)$, возникающие при импульсном фотовозбуждении, являются разностью двух спектров исследуемых образцов ЦО: $A(\lambda, t)$ при времени задержки $t$ и исходного спектра поглощения раствора ЦО без возбуждения $A_{0}(\lambda)$. Дифференциальные спектры $\Delta A(\lambda, t)$ были записаны в спектральном диапазоне 390-700 nm. Измеренные спектры подвергались коррекции, учитывающей дисперсию групповой скорости континуума, следуя процедуре, описанной в работе $[7,12]$. Эксперименты выполнялись при температуре $21^{\circ} \mathrm{C}$ в $0.5 \mathrm{~mm}$ оптической кювете.

Анализ временных характеристик транзиентных дифференциальных спектров осуществлялся с помощью кинетического моделирования на основе сингулярного разложения (SVD) матрицы полученных данных. Используемый подход предполагает рассмотрение всего массива экспериментальных значений $\Delta A(\lambda, t)$, где $\Delta A$, как отмечалось выше, отображает фотоиндуцированные изменения оптической плотности исследуемого образца (просветление и наведенное поглощение). Таким образом, при анализе результатов использовалась в основном схема, включающая в себя три последовательных интермедиата $(A, B, C)$ и стационарное состояние объекта исследования $G$, т.е. его состояние до возбуждения, а также после завершения всех фотоиндуцированных изменений при условии отсутствия необратимых фотохимических реакций. В результате последовательность нестационарных интермедиатов записывалась в виде

$$
A \stackrel{k_{1}}{\longrightarrow} B \stackrel{k_{2}}{\longrightarrow} C \stackrel{k_{3}}{\longrightarrow} G
$$

где $k_{i}$ - константы скоростей переходов между интермедиатами. Матрица $\Delta A(\lambda, t)$ представляется в виде

$$
\Delta A(\lambda, t)=\sum_{i=1}^{4} K_{i}(t) S_{i}(\lambda)
$$

где $S_{i}(\lambda)$ - спектры промежуточных состояний, $K_{i}(t)-$ заселенности этих состояний как функции времени. Глобальная экспоненциальная подгонка данных позволяет найти $k_{1}, k_{2}$ и $k_{3}$, при которых модельные данные максимально приближены к экспериментальным. В специально оговоренном случае последовательность релаксационных процессов записывалась в виде $A \stackrel{k_{1}}{\longrightarrow} B \stackrel{k_{2}}{\longrightarrow} G$, так как анализ экспериментальных данных полностью „укладывался“ в эту схему, и следовательно, после фотовозбуждения в объекте образовывались только два интермедиата.

\section{Результаты и обсуждение}

Представление и обсуждение результатов начнем с экспериментальных данных, полученных для окисленной формы ЦО. На рис. 2 и 3 приведены дифференциальные спектры поглощения $\Delta A$, измеренные в спектральном диапазоне от 390 до $560 \mathrm{~nm}$ при различных временных задержках. Некоторые спектры, полученные при одной и той же задержке, приводятся на обоих рисунках. В про-

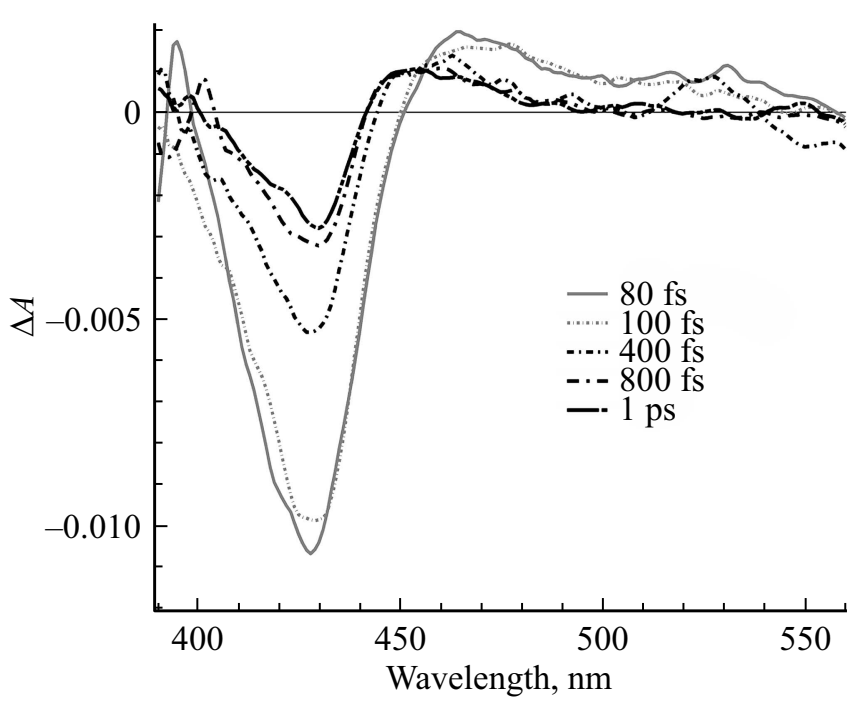

Рис. 2. Спектры дифференциального поглощения ЦО на временах задержки от $80 \mathrm{fs}$ до 1 ps при возбуждении импульсом при $600 \mathrm{~nm}$, длительностью $23 \mathrm{fs}$ и энергией $350 \mathrm{~nJ}$.

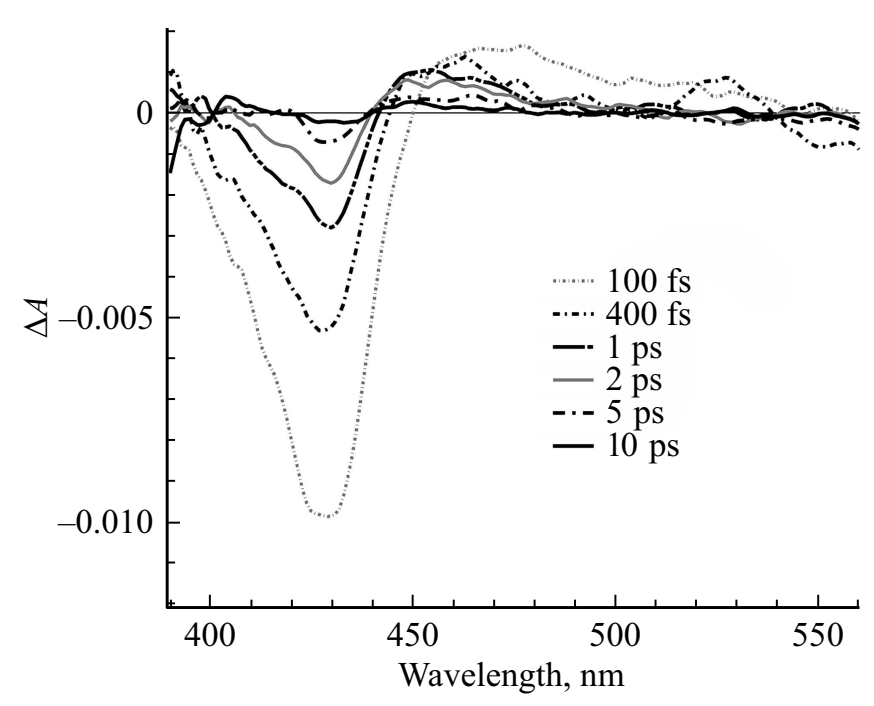

Рис. 3. Спектры дифференциального поглощения ЦО на временах задержки от $100 \mathrm{fs}$ до $10 \mathrm{ps}$ при возбуждении импульсом при $600 \mathrm{~nm}$, длительностью $23 \mathrm{fs}$ и энергией $350 \mathrm{~nJ}$. 
водимых экспериментах дифференциальные спектры измерялись в более широком спектральном диапазоне, а именно вплоть до $700 \mathrm{~nm}$. Обнаруженные спектральные изменения в спектральной области 625-700 nm мы не приводим, так как они были незначительны. В этой области не наблюдались максимумы в спектрах и происходило монотонное уменьшение сигналов фотоиндуцированного поглощения по мере продвижения в красную область спектра.

Наиболее значительные и характерные спектральные изменения заключались в просветлении полосы Соре и в появлении нового наведенного поглощения с длинноволновой и коротковолновой сторон спектра от этой полосы. Рассмотрение представленных на рис. 2 и 3 спектров указывает на сложную эволюцию фотоиндуцированных разностных спектров, что свидетельствует о наличии нескольких короткоживущих форм, которые формируют и трансформируют общий вид разностного спектра. Происходит смещение положений максимумов и изобестических точек в коротковолновую область спектра. Следует указать, что положение максимумов не соответствует их истинному положению в нестационарных спектрах интермедиатов, так как величина $\Delta A$ прямо пропорциональна разности коэффициентов экстинции ЦО в определенном возбужденном и основном состояниях в случае наведенного поглощения, а в случае просветления - разности коэффициентов поглощения в основном и возбужденном состояниях. Изобестические точки на спектрах расположены при длинах волн, на которых эти коэффициенты равны. Как указывалось в экспериментальной части, результаты измерений анализировались в рамках схемы, предполагающей наличие трех нестационарных интермедиатов $A, B$ и $C$. Аналогичный подход использовался нами в работе [7], в которой проводились исследования восстановленной формы ЦО. Следует специально отметить, что как для окисленной формы ЦО, так и для восстановленной обнаруживается присутствие пяти различных интермедиатов. Самый короткоживущий - длительностью $\leq 50 \mathrm{fs}$. Мы не рассматриваем его при глобальном анализе данных, который начинается с момента времени $80 \mathrm{fs}$. B наших экспериментальных условиях установить точный вид спектра, соответствующего этому интермедиату, и его длительности было невозможно. Необходимо указать, однако, что спектр поглощения данного интермедиата охватывал весь спектральный диапазон, в котором проводились измерения. Мы полагаем, что данное поглощение с длительностью $\leq 50 \mathrm{fs}$ относится к $S_{1}-S_{i}$-поглощению ЦО, т.е. поглощению из первого синглетного возбужденного состояния в более высокоэнергетические состояния. Подробное обсуждение данного спектра для восстановленной ЦО дано в работе [7]. Мы полагаем, что в случае окисленной формы ЦО мы имеем дело с полностью аналогичной ситуацией. Нами были также обнаружены спектрально-кинетические доказательства существования пятого $D$-интермедиата, самого долгоживущего, который проявляется при больших задержках,

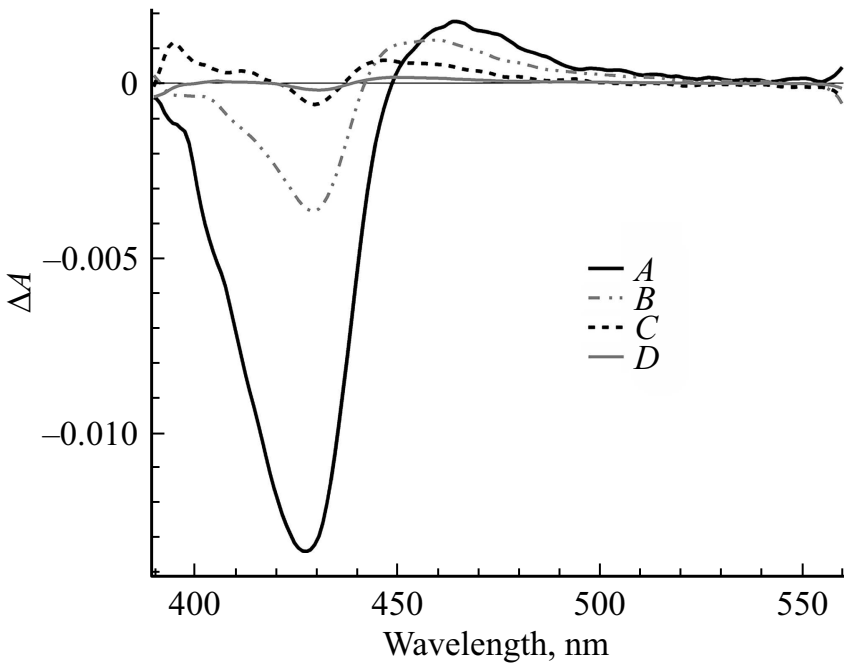

Рис. 4. Дифференциальные спектры интермедиатов $A, B$, $C$ и $D$. Константы скоростей дезактивации интермедиатов: $k_{1}=4.0 \pm 0.5 \mathrm{ps}^{-1}\left(\tau_{1}=250 f s\right), k_{2}=0.54 \pm 0.10 \mathrm{ps}^{-1}$ $\left(\tau_{2}=1.85 \mathrm{ps}\right)$ и $k_{3}=0.32 \pm 0.08 \mathrm{ps}^{-1}\left(\tau_{3}=3.12 \mathrm{ps}\right)$.

вплоть до 20 ps (рис. 4). Однако вклад этой спектральной формы в общую картину не превышает $2-3 \%$, и мы исключаем ее из детального рассмотрения и ограничиваемся лишь констатацией ее присутствия. Следует отметить, что интермедиат $D$ имеет ту же природу, что и интермедиат $C$, который будет обсуждаться далее.

На рис. 4 представлены дифференциальные спектры интермедиатов. Основной вопрос при анализе и интерпретации полученных спектров - какой вклад вносят в их формирование гем $a$ и гем $a_{3}$. Действительно, индивидуальный вклад каждого из гемов определяется главным образом двумя факторами: количеством фотонов, поглощенных каждым из них в отдельности, и соотношением величин коэффициентов экстинкции гема в основном и возбужденном состояниях. Как известно, что на длине волны возбуждения $\lambda=600 \mathrm{~nm}$ коэффициент экстинкции у гема $a$ примерно в пять раз больше, чем у гема $a_{3}[4,6]$. Следовательно, при анализе нестационарных спектров необходимо учитывать, что они формируются в результате вклада обоих гемов в примерном соотношении $5: 1$.

Приступим к анализу и интерпретации трех обнаруженных интермедиатов $A, B$ и $C$.

Интермедиат $A$. Дифференциальный спектр его наведенного поглощения охватывает весь видимый диапазон (рис. 4). Максимум в этом спектре расположен при $464 \mathrm{~nm}$, хоть необходимо отметить, что спектр носит достаточно „размытый характер“. Положение этого максимума отличается от истинного расположения в спектре поглощения интермедиата. Этот максимум располагается вблизи изобестической точки спектров поглощения интермедиата $A$ и ЦО в основном состоянии. Изобестическая точка расположена при 448-449 nm. Нахождение точного расположения максимума интерме- 
диата на данном этапе представляется исключительно сложным, так как оно требует специальных экспериментов, при которых все молекулы ЦО переводятся в это возбужденное состояние. Мы относим поглощение этого интермедиата к триплет-триплетному $\pi \pi^{*}$-поглощению ЦО, так как общий вид этого спектра очень близок к спектрам $T_{1}-T_{i}$-поглощения у металлопорфиринов, в том числе и у комплексов $\mathrm{Zn}$ и $\mathrm{Cd}$ с протопорфирином IX. Из-за очень короткого времени жизни интермедиата $A$ невозможно выполнить полное доказательство того, что это именно $T_{1}-T_{i}$-Поглощения, так как контрольный тест (тушение молекулярным кислородом этого возбужденного состояния) из-за очень короткого времени жизни интермедиата невозможен. Кроме того, у данного соединения, как и у всех гембелков, отсутствует фосфоресценция и флуоресценция. Мы уже отмечали, что имеем дело с результирующим спектром, однако мы полагаем, что основной вклад в наблюдаемый спектр интермедиата $A$ дает низкоспиновый гем $a$. Действительно, максимальное просветление наблюдается при $\lambda=428 \mathrm{~nm}$, что очень близко к значению максимума в индивидуальном спектре поглощения гема $a$, расположенного при $\lambda=426 \mathrm{~nm}[4]$.

Рассмотрим следующий кинетический интермедиат $B$ (рис. 4), время жизни которого составляет 1.85 ps. Максимум в дифференциальном спектре интермедиата $B$ претерпевает синий сдвиг на несколько нанометров в сравнении со спектром интермедиата $A$. Происходит также смещение изобестической точки. В результате они располагаются соответственно при 459 и $442 \mathrm{~nm}$. Следует отметить, что положение максимума в известной степени приблизительно, так как спектр интермедиата достаточно размыт. Максимальное просветление полосы Cоре наблюдается при $430 \mathrm{~nm}$. Таким образом, спектр интермедиата имеет главный максимум, расположенный в области полосы Соре. Точное положение максимума мы установить не можем, так как экспериментальные условия не позволяют перевести все молекулы ЦО в это состояние. Мы полагаем, что данный максимум располагается в $2-3 \mathrm{~nm}$ от изобестической точки $(\lambda=430 \mathrm{~nm})$, смещенный либо в красную, либо в синюю область спектра, а спектр интермедиата $B$ есть результат поглощения света ЦО в возбужденном $\pi d$-состоянии, т.е. в состоянии, при котором принадлежащий порфирину $\pi$-электрон претерпевает внутригемовый перенос на вакантное место на $d$-орбитале $\mathrm{Fe}$, т.е. образуется короткоживущий $\pi$-катион-радикал гема. Главный довод в пользу такой интерпретации основан на данных спектроскопии магнитного кругового дихроизма [6]. В этой работе в спектре МКД было зафиксировано поглощение с максимумом при $1600 \mathrm{~nm}$, которое авторы отнесли к $\pi-d$-переносу электрона. Кроме того, наличие низкоэнергетических состояний $\pi-d$-природы обнаружено для целого ряда низкоспиновых окисленных гембелков [13]. Уместно отметить также, что для ряда комплексов порфиринов с ионами переходных металлов спектры поглощения $\pi$-катион-радикалов хорошо извест-

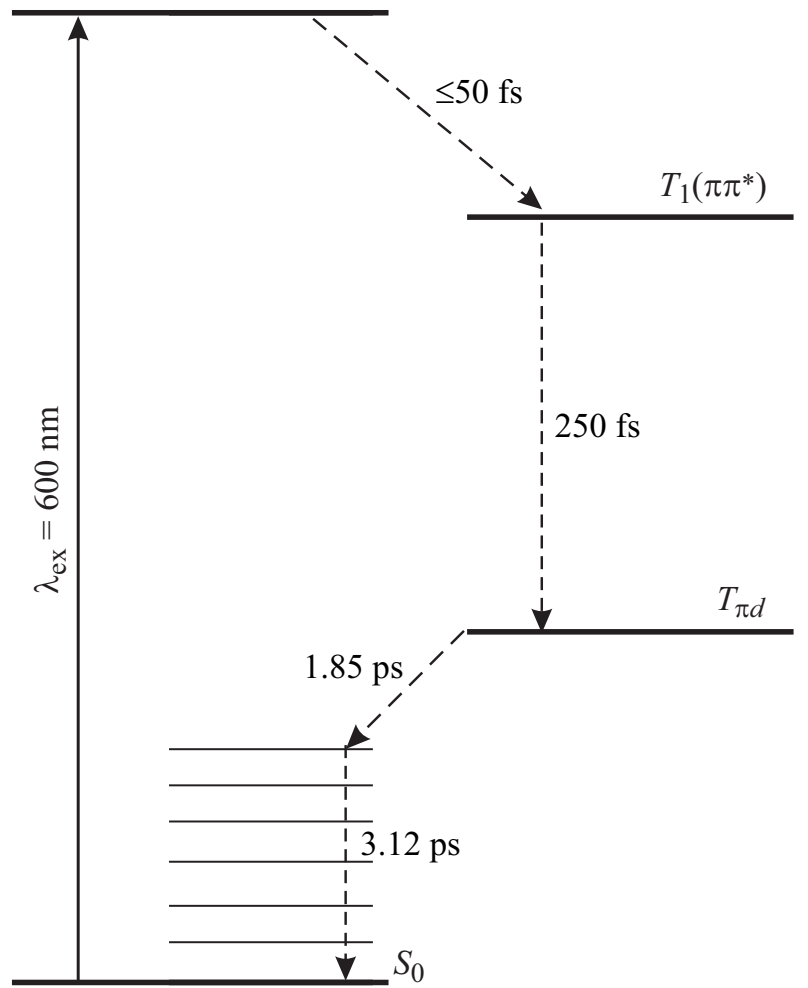

Рис. 5. Схема энергетических уровней и релаксационные процессы в геме $a$.

ны и близки по форме к обсуждаемому спектру $[14,15]$. Заселение состояния с $\pi-d$-переносом, мы полагаем, происходит из триплетного состояния, которое должно располагаться ниже синглетного $S_{1}$-состояния примерно на $\approx 3500 \mathrm{~cm}^{-1}$, что характерно для комплексов порфиринов с ионами переходных металлов. Отсутствие собственной фосфоресценции у гема $a$ и гема $a_{3}$, как и у любых других гемов, не позволяет непосредственно установить энергию состояния. Состояние с переносом заряда $(\pi-d)$ располагает энергией $\approx 6000 \mathrm{~cm}^{-1}$.

Интермедиат $C$. Данный интермедиат возникает из интермедиата $B$ и имеет время жизни $3.12 \mathrm{ps}$. Его спектр претерпевает „синий“ сдвиг по отношению к спектру интермедиата $B$. Максимум в его спектре и изобестическая точка форм $C$ и $G$ располагаются соответственно при 446 и $437 \mathrm{~nm}$. Максимальное просветление полосы Соре наблюдается при $430 \mathrm{~nm}$. Спектр интермедиата имеет симметричный $S$-образный „красный“ сдвиг по отношению к спектру поглощения в основном состоянии. Аналогичный характер спектров наблюдается практически во всех гембелках [16,17]. Данный спектр мы относим к поглощению окисленного гема $a_{3}$ в основном $G$-состоянии с избытком колебательной энергии, т.е. реализуется процесс „остывания“ гема - „heme-cooling“. Как правило, этот процесс носит биэкспоненциальный характер: основной вклад (90-95\%) дает быстрая составляющая (несколько ps); более медленная длится $\sim 20 \mathrm{ps}$. Для восстановленной 
формы ЦО биэкспоненциальный процесс наблюдался и обсуждался в работах $[7,18,19]$. В данной работе мы не исследовали длительную компоненту, на присутствие которой указывает интермедиат $D$, о котором шла речь ранее. Таким образом, для окисленной формы гема $a$ цитохром $c$ оксидазы $(\mathrm{Fe}(\mathrm{III}) a)$ наблюдается следующая последовательность релаксационных электронных и колебательных процессов. Электронные релаксационные процессы включают в себя три последовательные стадии (рис. 5). Первая, длительность которой $\approx 50 \mathrm{fs}$, представляет собой интеркомбинационный переход $S_{1}\left(\pi \pi^{*}\right) \rightarrow T_{1}\left(\pi \pi^{*}\right)$. Нельзя полностью исключить присутствие внутренней конверсии $S_{1} \rightarrow S_{0}$, так как мы не производили измерений квантового выхода интеркомбинационной конверсии. Однако известно, что интеркомбинационная конверсия для комплексов порфиринов с металлами $(\mathrm{Zn}, \mathrm{Cu}, \mathrm{Cd}, \mathrm{Pd})-$ основной канал дезактивации $S_{1}\left(\pi \pi^{*}\right)$-состояния [20,21]. Вторая, более длительная, стадия длительностью $250 \mathrm{fs}$ есть результат дезактивации триплетного $\pi \pi^{*}$-состояния в результате $T_{1}\left(\pi \pi^{*}\right) \rightarrow C T(\pi d)$-перехода. В результате этого заселяется $C T(\pi d)$-состояние, которое в свою очередь дезактивируется за счет перехода в основное $S_{0}(G)$-состояние. Длительность этого процесса $1.85 \mathrm{ps}$. После завершения трех процессов электронной релаксации молекула гема $a_{3}$ оказывается в основном состоянии с избытком колебательной энергии. Дальнейшая колебательная релаксация („остывание гема“) длится 3.12 ps. На рис. 5 представлена схема электронных возбужденных состояний и соответствующих релаксационных процессов. Представленная схема имеет упрощенный вид, так как не указаны состояния, связанные с возбуждением $d$-электронов, которые расположены выше $S_{1}$-состояния. Таким образом, в случае окисленного гема $a$ мы имеем картину, очень похожую на ту, которая наблюдается для восстановленной формы гема $a$ и которая была представлена нами в работе [7], с той лишь разницей, что в восстановленной форме гем $a$ из триплетного $\pi \pi^{*}$-состояния переходит в низколежащее $d d$-состояние, т.е. происходит перенос энергии с органической части молекулы на металл, в результате чего происходит $d-d$-переход и реализуется короткоживущее возбужденное состояние, связанное с возбуждением $d$-электрона Fe. В упомянутой нами работе [7] обсуждался также вопрос о релаксации энергии в восстановленной форме гема $a_{3}$, для которого несмотря на отсутствие прямых спектральных доказательств, предполагалось, что у него присутствуют лишь первые две стадии электронной релаксации, а самая длительная отсутствует.

В настоящей работе мы представляем результаты дополнительных исследований восстановленной формы цитохром $c$ оксидазы, выполненных в спектральной области $420 \pm 5 \mathrm{~nm}$, в которой поглощает в основном состоянии гем $a_{3}$ [5]. Было обнаружено, что в этой области кинетика спектральных изменений указывает на то, что число интермедиатов меньше на единицу.
Последовательность релаксационных процессов записывается в виде $A \stackrel{k_{1}}{\longrightarrow} B \stackrel{k_{2}}{\longrightarrow} G$. Биэкспоненциальная аппроксимация позволила для интермедитов $A$ и $B$ установить следующие времена жизни $182 \pm 8 \mathrm{fs}$ и $9.1 \pm 0.6 \mathrm{ps}$. Измерения были выполнены на длине волны возбуждения $\lambda=423 \mathrm{~nm}$, на которой наблюдается наибольший спектр. Эти кинетические характеристики относятся к интермедиатам восстановленной формы гема $a_{3}$. В связи с тем, что вся совокупность измерений была проведена в узком спектральном интервале, мы не располагаем дифференциальными спектрами интермедиатов гема $a_{3}$. Поэтому отнесение измеренных времен жизни к определенному возбужденному состоянию вызывает серьезные трудности. Окончательное нахождение этих спектров станет возможным в будущем после проведения специальных $f s$-измерений при возбуждении в области $\lambda \approx 420 \mathrm{~nm}$, т.е. при таких экспериментальных условиях, когда будет возбуждаться гем $a_{3}$. Вместе с тем мы полагаем, что интермедиат $A$ со временем $182 \mathrm{fs}$ представляет собой гем $a_{3}$ в триплетном $\pi \pi^{*}$-состоянии. На это указывает то, что очень близким временем жизни триплетного состояния обладает восстановленный гем $a$ $(200 \mathrm{fs})$ [7]. Интермедиат $B$ со временем жизни $9.1 \mathrm{ps}$, как и в случае интермедиата $C$ в геме $a$, представляет собой гем $a_{3}$ в основном состоянии с избытком колебательной энергии, т.е. мы наблюдаем колебательную релаксацию (heme cooling) длительностью $9.1 \mathrm{ps,} \mathrm{которая} \mathrm{близка} \mathrm{по}$ значению к длительности соответствующего процесса в геме $a(7.6 \mathrm{ps})$ [7]. Уместно отметить, что в работе [19] обсуждалась возможность того, что данный процесс в геме $a_{3}$ более длителен в сравнении с гемом $a$. Таким образом, в геме $a_{3}$ электронная релаксация включает два процесса: $S_{1} \rightarrow T_{1}(\tau \leq 50 \mathrm{fs})$ и $T_{1} \rightarrow S_{0}(182 \mathrm{fs})$. Следует отметить, что таким образом высокоспиновый гем $a_{3}$ в этом смысле аналогичен высокоспиновому дезоксигемоглобинному $\mathrm{Hb}$ [16]; у обоих гембелков $S=2$. Для этих двух гембелков характерно наличие в спектре поглощения полосы при $\approx 760-780 \mathrm{~nm}$, которая имеет перенос-зарядовую природу $\pi-d$. Однако заселение этого состояния в процессе дезактивации энергии $T_{1}\left(\pi \pi^{*}\right)$-состояния не происходит.

\section{Заключение}

Методом фемтосекундной абсорбционной спектроскопии исследован терминальный белок дыхательной цепи цитохром $c$ оксидаза. В результате обнаружены и изучены спектры фотоиндуцированного поглощения в спектральном диапазоне $390-560 \mathrm{~nm}$ и во временном интервале $80 \mathrm{fs}-20 \mathrm{ps}$. Несмотря на принципиальные трудности при анализе экспериментальных данных, обусловленные сильным перекрытием спектров поглощения гемов $a$ и $a_{3}$, мы полагаем, что в окисленной и восстановленной формах гема $a$ процессы электронной и колебательной релаксации в гемовом центре имеют общие черты. В них присутствует заселение и дезактивация 
триплетного $\pi \pi^{*}$-состояния, приводящие к заселению и последующей дезактивации более низкоэнергетического возбужденного электронного состояния, которое, однако, имеет у гема $a$ разную орбитальную природу в окисленной и восстановленной формах. После завершения стадий электронной релаксации молекула гема $a$ оказывается в основном состоянии с избытком колебательной энергии. Последующая колебательная релаксация длится несколько пикосекунд. В восстановленной форме гема $a_{3}$ реализуется электронная релаксация в результате последовательности двух процессов заселения и дезактивации триплетного $\pi \pi^{*}$-состояния с последующей колебательной релаксацией в основном состоянии.

\section{Благодарности}

Авторы благодарны А.А. Константинову и А.Ф. Чайковскому за проявленный интерес к работе и ценные замечания.

\section{Финансирование работы}

Работа выполнена при финансовой поддержке Государственной программы научных исследований Республики Беларусь „Фотоника, опто- и микроэлектроника 1.4.01“ (2016-2020), Российского фонда фундаментальных исследований (проект № 17-04-00160а), а также за счет субсидии, выделенной Федеральному центру химической физики им. Н.Н. Семенова РАН на выполнения государственного задания 0082-2019-0001 (рег. номер AAAA-A19-119012890064-7).

\section{Конфликт интересов}

Авторы заявляют, что у них нет конфликта интересов.

\section{Список литературы}

[1] Kaila V.R.I., Verkhovsky M.I., Wikström M. // Chem. Rev. 2010. V. 110. P. 7062.

[2] Yoshikawa S., Shimada A. // Chem. Rev. 2015. V. 115. P. 1936.

[3] Siletsky S.A., Konstantinov A.A. // Biochim. Biophys. Acta. 2012. V. 1817. N 4. P. 476.

[4] Vanneste W.H. // Biochemistry. 1966. V. 65. P. 838.

[5] Dyuba A.V., Vygodina T.V., Konstantinov A.A. // Biochemistry (Moscow). 2013. V. 78. N 12. P. 1358.

[6] Eglinton D.G., Johnson M.K., Thomson A.J., Gooding P.E., Greenwood C. // Biochem. J. 1980. V. 191. P. 319.

[7] Шелаев И.В., Гостев Ф.Е., Выгодина Т.В., Лепешкевич С.В., Джсагаров Б.М. // Химия высоких энергий. 2018. T. 52. № 1. C. 31; Shelaev I.V., Gostev F.E., Vygodina T.V., Lepeshkevich S.V., Dzhagarov B.M. // High Energy Chemistry. 2018. V. 52. N 1. P. 45.

[8] Vygodina T.V., Kirichenko A., Konstantinov A.A. // Biochim. Biophys. Acta. 2014. V. 1837. N 7. P. 1188.

[9] Fowler L.R., Richardson S.H., Hatefi Y. // Biochim. Biophys. Acta. 1962. V. 64. N 1. P. 170.
[10] Bukreev A., Mikhailov K., Shelaev I., Gostev F., Polevaya Yu., Tyurin V., Beletskaya I., Umansky S., Nadtochenko V. // J. Phys. Chem. A. 2016. V. 120. N 12. P. 1961.

[11] Ushakov E.N., Nadtochenko V.A., Gromov S.P., Vedernikov A.I., Lobova N.A., Alfimov M.V., Gostev F.E., Petrukhin A.N., Sarkisov O.M. // Chem. Phys. 2004. V. 298. P. 251.

[12] Shelaev I.V., Gostev F.E., Mamedov M.D., Sarkisov O.M., Nadtochenko V.N., Shuvalov V.A., Semenov A.Yu. // Biochim. Biophys. Acta. 2010. V. 1797. P. 1410.

[13] Makinen M.W., Churg A.K., Iron Porphyrins / Eds. Lever A.B.P., Gray H.B. Addison-Wesley, Reading, MA. 1983. Part 1. P. 141.

[14] Fuhrhop J.-H. Porphyrins and Metalloporphyrins / Ed. Smith K.M. Amsterdam: Elsevier/North-Holland Biomedical Press. 1976. Ch. 14. P. 593.

[15] Dolphin D., Addison A.W., Cairns M., Dinello R.K., Farrell N.P., James B.R., Paulson D.R., Welborn C. // Int. J. Quantum Chem. 1979. V. 16. P. 311.

[16] Kholodenko Y., Volk M., Gooding E., Hochstrasser R.M. // Chem. Phys. 2000. V. 259. P. 71.

[17] Lim M., Jackson T.A., Anfinrud P.A. // J. Phys. Chem. 1996. V. 100. N 29. P. 12043.

[18] Stoutland P.O., Lambry J.-C., Martin J.-L., Woodruff W.H. // J. Phys. Chem. 1991. V. 95. N 17. P. 6406.

[19] Джсагаров Б.М., Белянович Л.М., Константинов А.А., Руденок А.Н., Тихомиров С.А. // Докл. АН. 2000. T. 375. № 1. C. 112; Dzhagarov B.M., Belyanovich L.M., Konstantinov A.A., Rudenok A.N., Tikhomirov S.A. // Dokl. AN. 2000. V. 373. P. 53.

[20] Джсагаров Б.М., Чирвоный В.С., Гуринович Г.П. Лазерная пикосекундная спектроскопия и фотохимия биомолекул / Под ред. Летохова В.С. М.: Наука, 1987. C. 181; Dzhagarov B.M., Chirvonyi V.S., Gurinovich G.P. Laser Picosecond Spectroscopy and Photochemistry of Biomolecules / Ed. Letokhov V.S. Bristol and Philadelphia: Adam Hilger. 1987. Ch. 3. P. 137.

[21] Джсагаров Б.М., Гуринович Г.П., Новиченков В.Е., Салохиддинов К.И., Шульга А.М., Ганжса В.А. // Химическая физика. 1987. Т. 6. № 8. С. 1069; Dzhagarov B.M., Gurinovich G.P., Novichenkov V.E., Salokhiddinov K.I., Shulga A.M., Ganzha V.A. // Khimicheskaya Fizika. 1987. V. 6. N 8. P. 1069. 